\title{
On the possibility of a helium white dwarf donor in the presumed ultracompact binary $2 S$ 0918-549
}

\author{
J. J. M. in 't Zand ${ }^{1,2}$, A. Cumming ${ }^{3}$, M. V. van der Sluys ${ }^{2}$, F. Verbunt ${ }^{2}$, and O. R. Pols ${ }^{2}$ \\ 1 SRON National Institute for Space Research, Sorbonnelaan 2, 3584 CA Utrecht, The Netherlands \\ e-mail: jeanz@sron.nl \\ 2 Astronomical Institute, Utrecht University, PO Box 80000, 3508 TA Utrecht, The Netherlands \\ 3 Physics Department, McGill University, 3600 Rue University, Montreal, QC, H3A 2T8, Canada
}

Received 7 March 2005 / Accepted 19 June 2005

\begin{abstract}
S 0918-549 is a low-mass X-ray binary (LMXB) with a low optical to X-ray flux ratio. Probably it is an ultracompact binary with an orbital period shorter than $60 \mathrm{~min}$. Such binaries cannot harbor hydrogen rich donor stars. As with other (sometimes confirmed) ultracompact LMXBs, 2S 0918-549 is observed to have a high neon-to-oxygen abundance ratio (Juett et al. 2001, ApJ, 560, L59) which has been used to argue that the companion star is a CO or ONe white dwarf. However, type-I $\mathrm{X}$-ray bursts have been observed from several of these systems implying the presence of hydrogen or helium on the neutron star surface. In this paper, we argue that the companion star in 2S 0918-549 is a helium white dwarf. We first present a type-I X-ray burst from 2 S 0918-549 with a long duration of $40 \mathrm{~min}$. We show that this burst is naturally explained by accretion of pure helium at the inferred accretion rate of $\sim 0.01$ times the Eddington accretion rate. At higher accretion rates of $\sim 0.1$ Eddington, hydrogen is required to explain long duration bursts. However, at low rates the long duration is due to the large amount of helium that accumulates prior to the burst. We show that it is possible to form a helium white dwarf donor in an ultracompact binary if accretion starts during the first ascent of the giant branch, when the core is predominantly made of helium. Furthermore, this scenario naturally explains the high neon-to-oxgen ratio, without requiring a $\mathrm{CO}$ or ONe white dwarf companion. The only observational aspect of 2S 0918-549 that we cannot explain is the absence of helium lines in the optical spectrum. Model calculations of optical accretion disk spectra need to be carried out in order to obtain limits on the helium abundance.
\end{abstract}

Key words. X-rays: binaries - X-rays: bursts - X-rays: individuals: 2S 0918-549=4U 0919-54 - stars: evolution

\section{Introduction}

2S 0918-549 is a low-mass X-ray binary (LMXB) in which a Roche-lobe filling low-mass star orbits a neutron star and the liberation of gravitational energy of the in-falling matter produces X-rays. It is a particularly interesting example of a LMXB for three reasons. First, it has an optical to X-ray flux ratio which suggests that it is an ultracompact binary with an orbital period less than $\sim 60 \mathrm{~min}$ and a companion donor star that can only fit within the Roche lobe if it is hydrogen depleted (Juett et al. 2001; based on relations established by Van Paradijs $\&$ McClintock 1994). This was recently confirmed through optical spectroscopy which revealed the lack of lines from hydrogen and helium (Nelemans et al. 2004). It suggests that the companion star is a C-O or O-Ne white dwarf (cf., Nelson et al. 1986).

Second, it has an X-ray luminosity which is low for an active LMXB, namely less than or equal to $1 \%$ of the Eddington limit for a canonical $\left(1.4 M_{\odot}, 10 \mathrm{~km}\right.$ radius $)$ neutron star (Jonker et al. 2001).
Third, 2S 0918-549 appears to have an unusually high $\mathrm{Ne} / \mathrm{O}$ abundance ratio, a characteristic which it shares with three other non-pulsating LMXBs out of 56 cases investigated by Juett et al. (2001). Juett et al. propose that this is related to a degenerate donor in the suggested ultracompact nature of the binary. This proposition is supported by detections of orbital periods in two of the other three (18 min in 4U 1543-624, see Wang \& Chakrabarty 2004; $21 \mathrm{~min}$ in 4U 1850-087, see Homer et al. 1996), although those detections need corroboration since they were made only once in each case. Recently, it was observed for these high $\mathrm{Ne} / \mathrm{O}$ systems that the $\mathrm{Ne} / \mathrm{O}$ ratio changed while for another previously not measured ultracompact system the Ne/O ratio was not high (Juett \& Chakrabarty 2003 and 2005). This indicates that the interpretation of $\mathrm{Ne} / \mathrm{O}$ in terms of donor evolutionary status is less straightforward and is possibly biased by ionization effects. For 2S 0918-549, the $\mathrm{Ne} / \mathrm{O}$ ratio was found to be 2.4 times the solar value from Chandra-LETGS and XMM-Newton observations, while it was 5 times the solar value during the ASCA measurement (when the continuum flux was an order of magnitude larger). It should 
be noted that the ASCA measurement was performed at a significantly worse spectral resolution.

Three of the four LMXBs with a possibly high Ne/O-ratio exhibit sporadic type-I X-ray bursts which are due to thermonuclear flashes in the upper layers of a neutron star. Four have been reported from 4U 0614+091 (Swank et al. 1978; Brandt et al. 1992, 1993a, 1993b), four from 4U 1850-087 (Swank et al. 1976; Cominsky et al. 1977; Hoffman et al. 1980; Cominsky 1981) and three from 2S 0918-549 (Jonker et al. 2001; Cornelisse et al. 2002; Galloway et al. 2005). We here report four additional bursts from the latter. Additional bursts for the other systems were also detected, particularly with HETEII (see for instance Nakagawa et al. 2004), but these are not published in detail yet. The one source never seen bursting is $4 \mathrm{U}$ 1543-624.

As noted by Juett et al. (2003) and Nelemans et al. (2004), there is a puzzling contradiction between the characteristics (or mere presence) of these bursts and the suggested hydrogen or helium depletion in the donor stars. The bursts detected from these systems last between 10 and a few hundred seconds, suggesting a high hydrogen and helium content in the flash fuel. The question is: where does the hydrogen and helium come from if not from the donor star? The problem is more severe for hydrogen than for helium because in an evolved donor star like in an ultracompact system the hydrogen can reside only in the outer layers that were lost being outside the Roche lobe. Juett et al. and Nelemans et al. suggest that spallation of accreted elements may be important (e.g., Bildsten et al. 1992). However, it is not trivial to invoke spallation. Spallation requires hydrogen nuclei (protons) to bombard the higher-up Coulomb-stopped heavy nuclei and create lighter elements, but the problem is that protons are in short supply. A definite assessment of the viability of this process needs to come from new calculations that also take into account non-radial accretion (e.g., Bildsten et al. 2003) and high metal abundances.

In this paper we present (in Sects. 2 and 3) measurements of an extraordinary burst from 2S 0918-549 which was detected with the Wide Field Cameras (WFCs; Jager et al. 1997) on board BeppoSAX (Boella et al. 1997a). It is the longest of all bursts observed from any (presumed) ultracompact, lasting over half an hour. Commonly, long burst durations (for example, bursts from the regular burster GS 1826-24; Ubertini et al. 1999; Galloway et al. 2004) are explained by a high hydrogen fuel content. The protons are captured by the ashes of unstable helium burning and initiate a relatively slow beta decay process (the rp process) that is responsible for the burst longevity (e.g., Fujimoto et al. 1981). However, at the low accretion rate of $\sim 1 \%$ Eddington appropriate for $2 \mathrm{~S}$ 0918-549, the conditions at the time of ignition of the flash are different. Because the fuel accumulates slowly, any hydrogen has time to stably burn away, leaving a thick layer of helium which ignites and burns in a long duration and energetic burst. In fact, given the likely ultracompact nature of this source, we argue that the long duration burst is due to accretion of pure helium from a helium white dwarf companion. As we show in Sect. 5.1, 2S 0918-549 provides the rarely seen circumstances for a long duration helium flash to be possible, being a persistent X-ray source at a fairly low mass accretion rate. We investigate in Sect. 5.2
Table 1. List of X-ray bursts from 2 S 0918-549. Bursts 2, 3 and 7 have been published before. Values between parentheses represent uncertainties in the last digit.

\begin{tabular}{|c|c|c|c|c|c|}
\hline \multirow[t]{2}{*}{ No. } & Date & Instr. & $\begin{array}{l}\text { Peak } \\
\text { flux }\end{array}$ & $\begin{array}{l}\text { Persistent } \\
\text { flux }\end{array}$ & \multirow{2}{*}{$\begin{array}{l}\tau \\
(\mathrm{s})\end{array}$} \\
\hline & & & \multicolumn{2}{|c|}{$\left(\right.$ Crab units $\left.^{\star}\right)$} & \\
\hline 1 & 1996 Oct. 1 & WFC & $3.7(3)$ & $0.0048(5)$ & $117(2)$ \\
\hline $2^{\dagger}$ & 1999 Jun. 10 & WFC & $3.3(3)$ & $0.0037(5)$ & 29(4) \\
\hline $3^{\ddagger}$ & 2000 May 12 & PCA & $>3.2$ & $<0.0146$ & $8.95(5)$ \\
\hline 4 & 2001 May 18 & WFC & $2.5(3)$ & $0.0094(5)$ & $5.8(4)$ \\
\hline 5 & 2001 Sep. 29 & ASM & $2.9(1)$ & $0.0061(7)^{\mathbb{I}}$ & $25(3)$ \\
\hline 6 & 2003 Aug. 5 & ASM & 2.1(1) & $0.0080(8)^{\mathscr{I}}$ & $22(10)^{x}$ \\
\hline $7^{*}$ & 2004 Jun. 18 & PCA & $0.26(1)$ & $0.0126(3)$ & $12.5(5)$ \\
\hline
\end{tabular}

* For a burst peak spectrum as determined in this paper for the first burst, one Crab unit translates to a bolometric flux of $2.7 \times$ $10^{-8} \mathrm{erg} \mathrm{cm}^{-2} \mathrm{~s}^{-1}$. For the persistent flux, one may adhere to a $2-10$ $\mathrm{keV}$ flux of $2.0 \times 10^{-8} \mathrm{erg} \mathrm{cm}^{-2} \mathrm{~s}^{-1}$ (the bolometric correction is less certain in that case).

$\dagger$ Cornelisse et al. (2002). This burst was erroneously dated four days earlier in that paper.

Jonker et al. (2001).

* Galloway et al. (2005) this paper mentioned 2 more faint bursts from 2S 0918-549, but the data do not allow confirmation as type-I X-ray bursts.

IIThese are 14-d averages.

${ }^{\times}$This burst was only partly observed. The observation stopped $18 \mathrm{~s}$ after burst onset.

evolutionary paths to arrive at the implied helium-rich donor star and find a likely path leading to a helium white dwarf. What is more, this star is predicted to have a Ne/O overabundance ratio which confirms the observations. Thus, a model in which the companion star is a helium white dwarf explains many peculiar details about 2S 0918-549. One detail which is not explained concerns the lack of helium lines in the optical spectrum.

\section{Synopsis of X-ray bursts from $2 S$ 0918-549}

Thus far three X-ray bursts were reported from 2S 0918-549, by Jonker et al. (2001), Cornelisse et al. (2002) and Galloway et al. (2005). The first two bursts have similar bolometric peak fluxes of 8.8 and $9.4 \times 10^{-8} \mathrm{erg} \mathrm{cm}^{-2} \mathrm{~s}^{-1}$, but the decay times differ by a factor of 3 (see Table 1). The third burst as identified by Galloway is an order of magnitude fainter. We carried out archival searches in BeppoSAX/WFC data (net exposure 9.2 Ms), RXTE/ASM (2.6 Ms for an effective exposure time of $70 \mathrm{~s}$ per dwell), and RXTE/PCA data ( $300 \mathrm{ks}$, including data from AO9), and found 2 more bursts in ASM and 2 in WFC data. No reports of bursts were made from observations with Einstein, EXOSAT, ROSAT, ASCA, BeppoSAX, Chandra and XMM-Newton within a total of approximately $170 \mathrm{ks}$. We derive an average burst rate (from simple division of the exposure time by the number of bursts) of once every 20 days, but note that the WFCs and ASM are not sensitive enough to detect the fainter bursts. Based on PCA data alone, the average burst rate is once every $2 \pm 1 \mathrm{~d}$. Table 1 reports the main characteristics of all 7 bursts. 


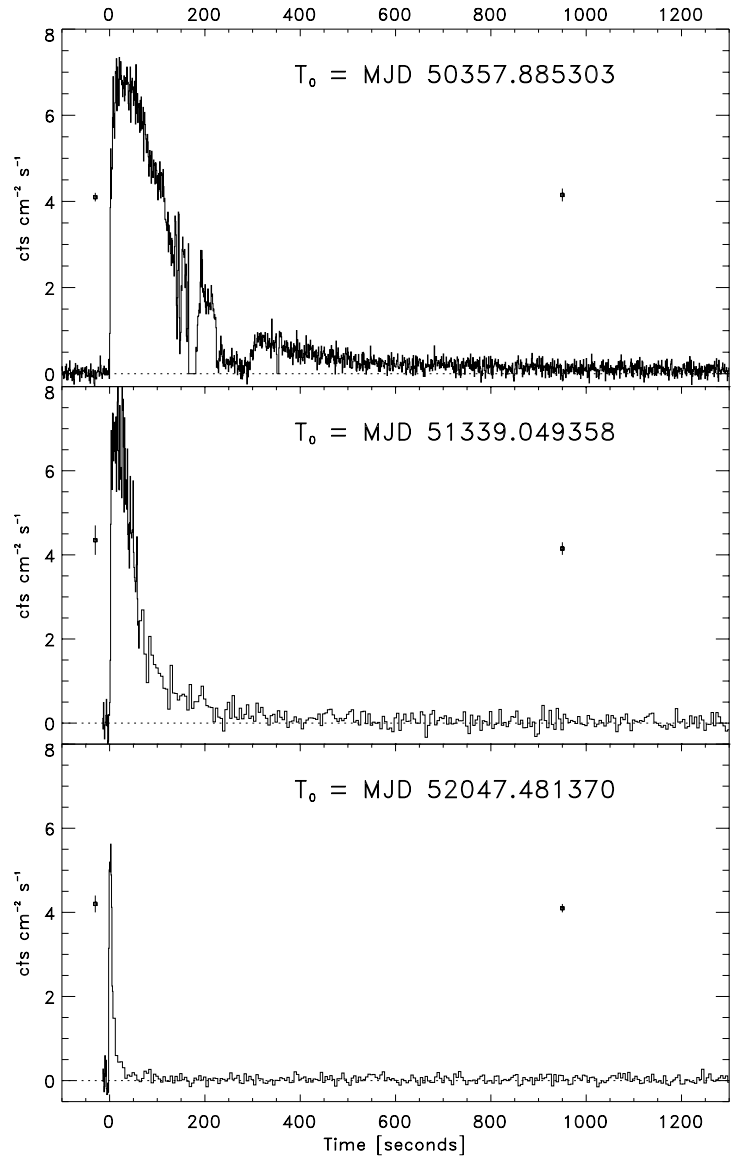

Fig. 1. 2-28 keV light curves of the three WFC-detected bursts at varying time resolution. Typical error bars are indicated at the left and right of each panel.

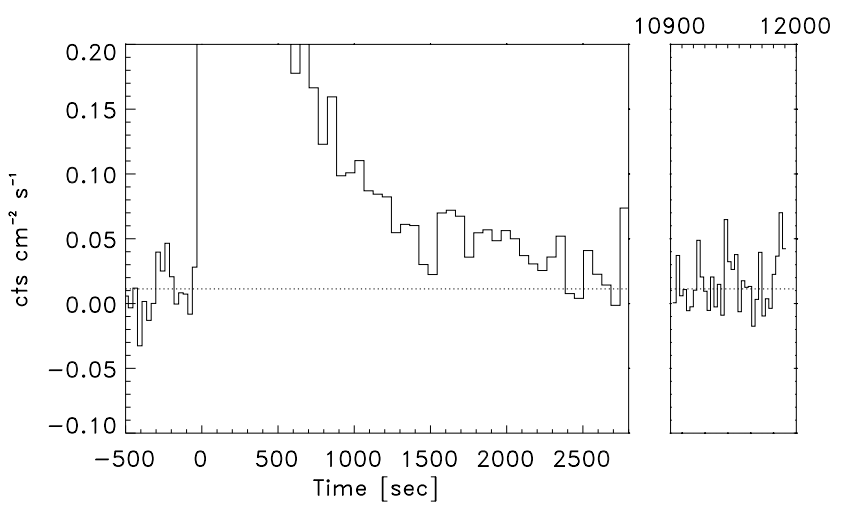

Fig. 2. 2-28 keV light curve of the long burst, zooming in at low flux levels and with a time resolution of $30 \mathrm{~s}$. The dotted line indicates the out-of-burst persistent flux level. There are no data during times between the two panels.

Except for the last, all bursts are fairly bright with bolometric peak fluxes that translate to between $6 \times 10^{-8}$ and $10^{-7} \mathrm{erg} \mathrm{cm}^{-2} \mathrm{~s}^{-1}$. Cornelisse et al. (2002), equalizing the peak flux of the second burst to the Eddington limit of a hydrogenrich photosphere, derive a distance of $4.2 \mathrm{kpc}$ with an uncertainty of $30 \%$. This is in contrast to an earlier distance estimate, from optical measurements, of $15 \mathrm{kpc}$ by Chevalier \& Ilovaisky (1987) which is derived assuming the optical

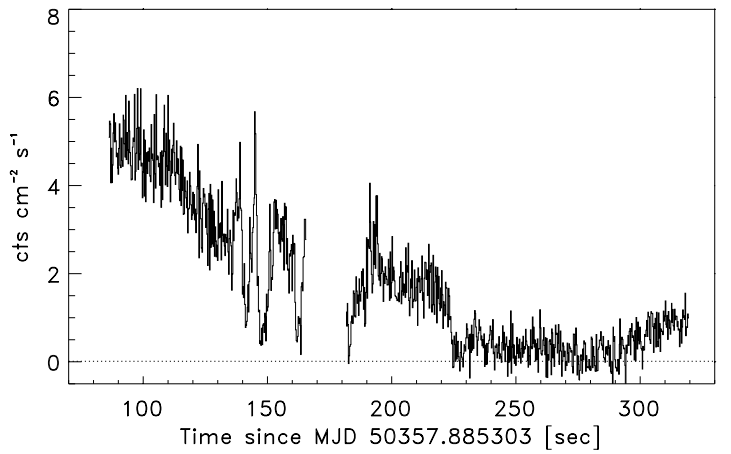

Fig. 3. 2-28 keV light curve of the long burst at a time resolution of $0.25 \mathrm{~s}$, zooming in on the period of strong variability.

counterpart to be as luminous as in other LMXBs. Therefore, the counterpart must be considerably sub luminous which is indicative of a small accretion disk and short orbital period of at most 60 min (Juett et al. 2001).

In Fig. 1 the three bursts detected with the WFC are plotted on identical scales. This illustrates the diversity of the bursts. In particular it illustrates the longevity of the first burst. In Fig. 2 the flux scale is blown up for this burst and it is clear that it persists for at least approximately $2500 \mathrm{~s}$. The second burst lasts 10 times as short, the third burst nearly 100 times as short, the bright PCA-detected burst about 25 times (Jonker et al. 2001).

\section{Analysis of the long burst}

The long burst started on October 1, 1996, at 21:14:51 UT, rose to peak levels within $1 \mathrm{~s}$ and carried on for approximately 40 min before it disappeared in the background noise (at a level $\approx 350$ times below the burst peak; see Fig. 2). The e-folding decay time over the first $200 \mathrm{~s}$ is $117 \pm 2 \mathrm{~s}$. This is the longest decay time of all 2427 type-I X-ray bursts measured with the WFCs that are not superbursts (In 't Zand et al. 2004b; Kuulkers 2004), except for one burst from SLX 1737-282 (In 't Zand et al. 2002) which exhibited a decay time of $600 \mathrm{~s}$.

The off-axis angle of 2S 0918-549 in the WFC field of view during the long burst was near to optimum. The source illuminated $90 \%$ of the available sensitive detector area. However, the observation was plagued by high telemetry rates due to a bright source within the concurrent narrow-field instruments, which resulted in sporadic WFC data drop outs. Two drop outs occurred during the long X-ray burst, from 165 to $180 \mathrm{~s}$ after burst onset and from 350 to $354 \mathrm{~s}$.

There are two more conspicuous features in the time profile of the long burst, see Fig. 3: two minutes after the burst onset a $30 \mathrm{~s}$ period of strong variability is observed, with dips and peaks that grow above the flux before that. Two minutes later the flux suddenly (within $2 \mathrm{~s}$ ) decreases by a factor of about four. Subsequently it remains on a decaying track. After a little over $70 \mathrm{~s}$ it rises back to the extrapolated pre-drop downward trend and continues its decay. Since several data drop outs occurred during this observation we checked whether this drop in flux could be due to telemetry overflow. We studied the flux history of another bright source in the field of view, Vela X-1, and that of the remaining background. These showed no flux 


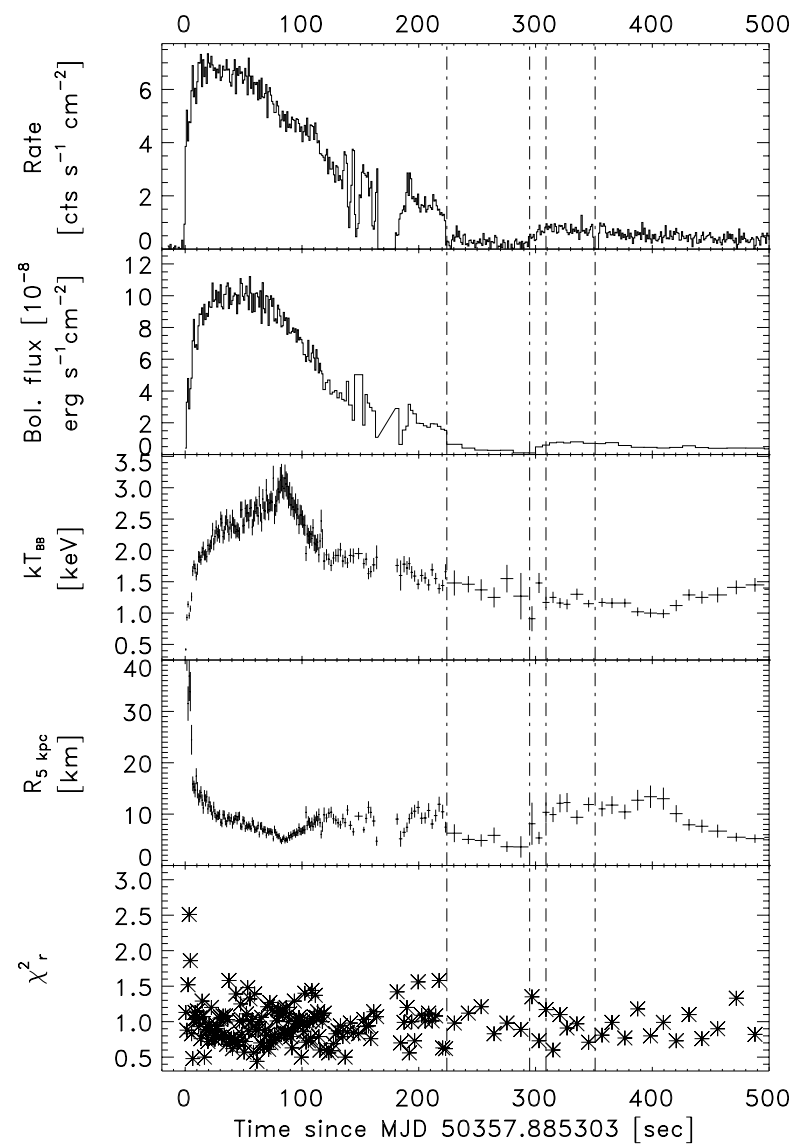

Fig. 4. a) Time history of observed photon flux. b) Bolometric flux of modeled black body radiation. c) Color temperature of black body radiation. d) Sphere-equivalent radius of bb radiation for a distance of $5 \mathrm{kpc}$ (the first data point of $208 \pm 25 \mathrm{~km}$ is outside the plot borders). e) $\chi_{v}^{2}$ of fits. The vertical lines indicate time intervals for further spectroscopy of the dip (see Fig. 5).

decrease whatsoever, in contrast to during the data drop out periods. We conclude that the flux decrease during the burst is genuinely associated with 2 S 0918-549.

We modeled the spectrum of the long burst with black body radiation and present the results in Fig. 4 . The burst starts with a strong photospheric radius expansion phase which ends after a few seconds. Subsequently the bolometric flux remains at a level of about $10^{-7} \mathrm{erg} \mathrm{cm}^{-2} \mathrm{~s}^{-1}$ for one minute during which the temperature rises and the radius shows a slow instead of fast decrease. This suggests that the photosphere is continuously injected with fresh (radiation) energy since the fallback time of the atmosphere is much shorter. Thereafter the burst decays in an ordinary fashion for about 3 min with decreasing temperatures and constant radii. This suggests cooling by a fairly thick layer. Four minutes after the burst onset the flux suddenly (within $2 \mathrm{~s}$ ) drops by a factor of 4 . The flux remains low for $73 \mathrm{~s}$ after which it slowly rises during $20 \mathrm{~s}$ and resumes the pre-drop decay. The e-folding decay time then is $236 \pm 11 \mathrm{~s}$. The cause of the dip can be modeled in two ways: either through a variation of $N_{\mathrm{H}}\left(\chi_{v}^{2}=0.85\right.$ for $v=78$ for simultaneously fitting the 3 spectra of the dip [exposure time $71 \mathrm{~s}$ ], the rise out of the dip [14 s], and a period afterwards [41 s]) or through a variation of

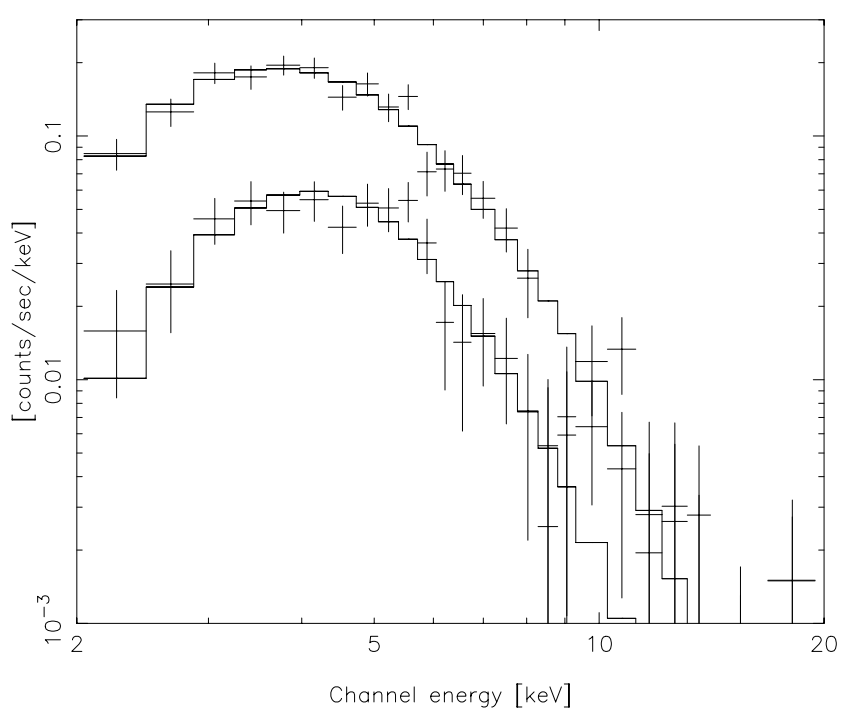

Fig. 5. Spectra of the $71 \mathrm{~s}$ lower-flux interval (lower spectrum; first interval between vertical lines in Fig. 4) and the $41 \mathrm{~s}$ interval after the dip (upper spectrum; last interval in Fig. 4). The crosses indicate the measurements (vertical lengths indicate $1 \sigma$ error intervals; histograms indicate the fitted model with free $N_{\mathrm{H}}$ and fixed radius).

the emission area $\left(\chi_{v}^{2}=0.82\right.$ for $\left.v=78\right)$. Figure 4 shows the results of the latter model. The implied reduction in emission area is a factor of 6 (or 2.5 in radius). When modeled through an increase of the absorption, the implied maximum column density is $N_{\mathrm{H}}=8.3_{-2.5}^{+2.9} \times 10^{22} \mathrm{~cm}^{-2}$ (90\% confidence; compare with $N_{\mathrm{H}}=4.2 \times 10^{-2 .} \mathrm{cm}^{-2}$ outside the dip).

If one equalizes the peak bolometric flux to the Eddington limit of a canonical $1.4 M_{\odot}$ neutron star, the implied distance is $4.1 \mathrm{kpc}$ for a hydrogen-rich photosphere and $5.4 \mathrm{kpc}$ if it is hydrogen-poor.

Ignoring the behavior of the source during the dip and data drop outs, the total bolometric energy output for a distance of $5.4 \mathrm{kpc}$ is estimated to be $(0.9 \pm 0.4) \times 10^{41} \mathrm{erg}$. This is a factor of at least 3 smaller than any of the 13 superbursts observed sofar (cf. Kuulkers 2004 and In 't Zand et al. 2004a) but similar to the most energetic non-super X-ray burst (from SLX 1737-282, In 't Zand et al. 2002).

What is the cause of the 1.2 min drop during the decay phase? The profile of the flux history looks strikingly similar to a partial eclipse. However, this is inconceivable because 1) the radiating surface is so small that a partial eclipse seems very unlikely, and 2) eclipses have never been seen in 2S 0918-549 down to very good limits (e.g., Juett \& Chakrabarty 2003).

The effect looks a bit like the few-second long dip seen in the superburst from 4U 1820-303 (Strohmayer \& Brown 2002). There it was attributed to the combination of photospheric radius expansion and a clean sweep of the normally $\mathrm{X}$-radiating inner accretion disk. This explanation is inconsistent with the temperature evolution seen in 2S 0918-549. Still, it seems likely that the dip is related to a perhaps more moderate change in the accretion flow geometry induced by the radiation pressure of the luminous flash. An indication of that is provided by the strong variability in the minute before the dip. 


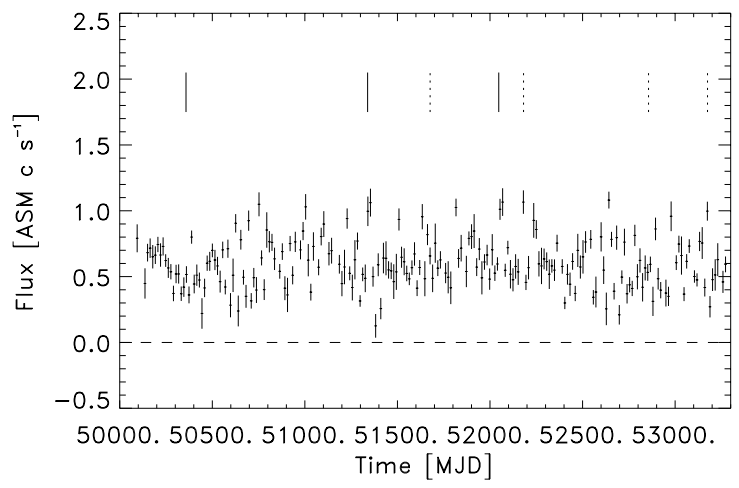

Fig. 6. RXTE/ASM 2-12 keV light curve with a bin time of $14 \mathrm{~d}$ and eliminating all data points from SSC3 or with an error in excess of $0.15 \mathrm{c} \mathrm{s}^{-1}$. The vertical lines in the top indicate the times when bursts were detected (solid lines WFC, dashed lines RXTE PCA (1st and last) and ASM).

\section{Flux history of persistent emission}

Given the wide variety of burst durations, it is of interest to test whether there is a connection with a varying mass accretion rate. Therefore, we studied data relating to the persistent flux history. 2S 0918-549 is quite faint for monitoring devices such as the WFC or the RXTE All-Sky Monitor (Levine et al. 1996). This hampers accurate measurements on time scales below a few weeks. In Fig. 6, the 2-12 keV flux history is plotted as measured with the RXTE ASM with a $14 \mathrm{~d}$ binning time. On that time scale the flux ranges between 0.3 and $1.0 \mathrm{ASM} \mathrm{c} \mathrm{s}^{-1}$ which, for a Crab-like spectrum, translates to a $2-10 \mathrm{keV}$ flux of $(1-3) \times 10^{-10} \mathrm{erg} \mathrm{cm}^{-2} \mathrm{~s}^{-1}$. We note that no flares were observed on shorter time scales above a limit of roughly $0.1 \mathrm{Crab}$ units, except for the bursts. In the same plot the times of the 7 bursts are indicated. The long (first) burst distinguishes itself from the other bursts by occurring during a somewhat tranquil low state of the source.

There has been one broad-band X-ray measurement of the source, by the BeppoSAX Narrow Field Instruments (Boella et al. 1997a) on 1998 April 22. Broad-band coverage was obtained through the Low-Energy Concentrator Spectrometer (LECS; Parmar et al. 1997; 0.1-3.0 keV; 16 ks exposure time), the Medium Energy Concentrator Spectrometer (MECS; Boella et al. 1997b; 1.6-10.0 keV; $28 \mathrm{ks)} \mathrm{and} \mathrm{the} \mathrm{Phoswhich}$ Detector System (PDS; Frontera et al. 1997; 15-200 keV; $12 \mathrm{ks})$. The LECS and MECS provided imaging data, while the PDS operated with a collimator that rocked between on-source and background pointings $240^{\prime}$ from the source position. We verified that no bright $\mathrm{X}$-ray source was contained in the background pointings.

We employed standard extraction and data analysis techniques (e.g., In 't Zand et al. 1999) and restricted further analysis to those photon energies where there is a significant detection (extending from 0.3 to $120 \mathrm{keV}$ ). The LECS and MECS extraction radii were $4^{\prime}$, a LECS/MECS and PDS/MECS normalization factor was left free during spectral fits, LECS and MECS background spectra were determined from independent long observations on empty fields, and no systematic uncertainty was included. Various models were tested against the
Table 2. Spectral parameters of acceptable model fits to the NFI spectrum. $\Gamma$ is the photon index. Errors are for $90 \%$ confidence.

\begin{tabular}{ll}
\hline \hline Model & power law + black body \\
$N_{\mathrm{H}}$ & $(3.1 \pm 0.3) \times 10^{21}$ \\
bb $k T$ & $0.51 \pm 0.03 \mathrm{keV}$ \\
$\Gamma$ & $2.07 \pm 0.05$ \\
$\chi_{v}^{2}$ & $1.21(133 \mathrm{dof})$ \\
Unabs. $0.1-200 \mathrm{keV}$ flux & $(6.4 \pm 0.5) \times 10^{-10} \mathrm{erg} \mathrm{cm}^{-2} \mathrm{~s}^{-1}$ \\
Abs. 2-10 keV flux & $(1.37 \pm 0.02) \times 10^{-10} \mathrm{erg} \mathrm{cm}^{-2} \mathrm{~s}^{-1}$ \\
\hline Model & comptt + black body \\
$N_{\mathrm{H}}$ & $(2.8 \pm 0.3) \times 10^{21}$ \\
bb $k T$ & $0.51 \pm 0.03 \mathrm{keV}$ \\
$k T_{\text {plasma }}$ & $34_{-17}^{+110} \mathrm{keV}$ \\
$\tau$ (spherical geometry) & $1 \pm 0.5$ \\
$\chi_{\mathrm{r}}^{2}$ & $1.20(131 \mathrm{dof})$ \\
Unabs. 0.1-200 keV flux & $(5.5 \pm 0.5) \times 10^{-10} \mathrm{erg} \mathrm{cm}^{-2} \mathrm{~s}^{-1}$ \\
Abs. 2-10 keV flux & $(1.37 \pm 0.02) \times 10^{-10} \mathrm{erg} \mathrm{cm}^{-2} \mathrm{~s}^{-1}$ \\
\hline
\end{tabular}

data; two have a satisfactory result. These are presented in Table 2. The power-law fit is shown in Fig. 7. We tested a pure Comptonized model (model comptt in XSPEC; Arnaud 1996; Titarchuk 1994; Hua \& Titarchuk 1995; Titarchuk \& Lyubarskij 1995), and a simple power law. Both models were absorbed (following the model by Morrison \& McCammon 1983) and a black body component was included describing the $0.7 \mathrm{keV}$ feature discussed by Juett et al. (2001). The fit results are consistent with those obtained by Juett \& Chakrabarty (2003). The (absorbed) $2-10 \mathrm{keV}$ flux is consistent with the ASM measurements. The unabsorbed $0.1-200 \mathrm{keV}$ flux is the same in both cases.

The $0.1-200 \mathrm{keV}$ flux of $(6.0 \pm 0.5) \times 10^{-10} \mathrm{erg} \mathrm{cm}^{-2} \mathrm{~s}^{-1}$ is less than $1 \%$ of the bolometric burst peak flux of $(1.0 \pm$ $0.1) \times 10^{-7} \mathrm{erg} \mathrm{cm}^{-2} \mathrm{~s}^{-1}$ which is thought to be the Eddington limit. For a distance of 4.1-5.4 kpc the $0.1-200 \mathrm{keV}$ luminosity is $(1.2-2.1) \times 10^{36} \mathrm{erg} \mathrm{s}^{-1}$. The ASM light curve suggests that the source never becomes brighter than roughly twice this value, on time scales of weeks. The $2-10 \mathrm{keV}$ absorbed flux is also consistent with similar measurements since the 1970s as compiled by Juett et al. (2003) which range between 0.9 and $2.7 \times 10^{-10} \mathrm{erg} \mathrm{cm}^{-2} \mathrm{~s}^{-1}$, except for an ASCA measurement on 1995 May 2 (MJD 49 839; 17 months before the first burst) when the flux was $7.0 \times 10^{-10} \mathrm{erg} \mathrm{cm}^{-2} \mathrm{~s}^{-1}$. We note that an analysis of the near-to-continuous BATSE data set on 2S 0918549 by Harmon et al. (2004) does not discuss a peak in 1995.

2S 0918-549 has never been seen in an off state, despite extensive coverage since the early 1970s. It is therefore not an X-ray transient. The reason that it is persistent while the luminosity is rather low is possibly related to the presumed ultracompact nature. A smaller orbit generally implies a smaller accretion disk. Therefore, the disk will remain completely photo-ionized at lower accretion rates and the accretion will sustain all the way to the neutron star rather than turn off due to an accretion disk instability (White et al. 1984; van Paradijs 1996; Deloye \& Bildsten 2003). 


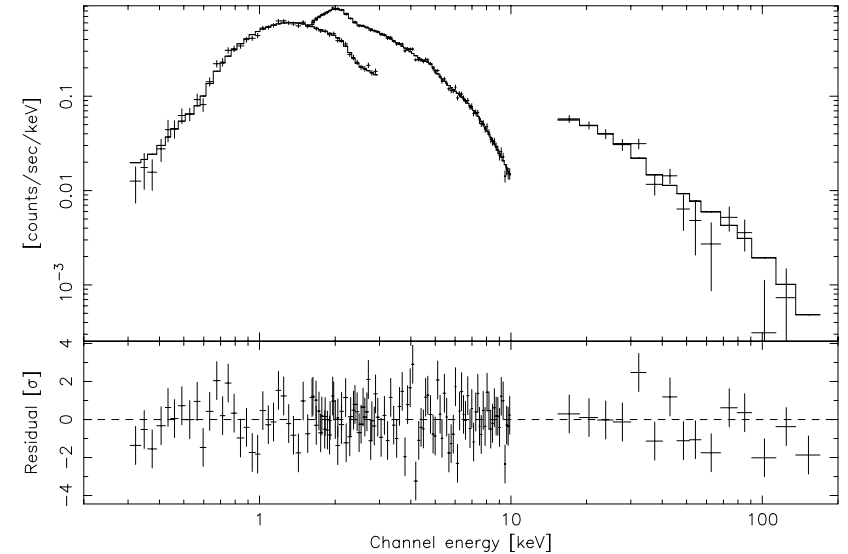

Fig. 7. Top panel: from left to right LECS, MECS and PDS spectrum (crosses with vertical lines depicting $1 \sigma$ error bars) and best fit result for power law and black body model (histogram). Bottom panel: fit residuals.

\section{Discussion}

\subsection{Short and long helium bursts}

In general, the longevity of an X-ray burst is determined by the duration of the nuclear burning, and by the thickness and composition (through thermal conductivity) of the layer where the burning deposits heat. Whereas helium and carbon burn very rapidly, hydrogen burning involves slow beta decays, and so can prolong the energy generation. Slow hydrogen burning via the rp-process (Wallace \& Woosley 1981) is believed to power the minutes long tails of bursts from GS 1826-24 (Galloway et al. 2004). For accretion rates $\sim 0.1$ times Eddington, appropriate for most X-ray burst sources, this has led to the identification of "short" duration bursts $(\sim 10 \mathrm{~s})$ with heliumdominated flashes, and long duration $(\sim 100 \mathrm{~s})$ bursts with hydrogen-dominated flashes. In this picture, the long duration burst from 2S 0918-549 is difficult to explain because we expect the companion to be hydrogen deficient. However, at low accretion rates, long bursts can arise because of very thick fuel layers that accumulate between bursts. These thick layers have a long cooling time, leading to long burst durations. In this section, we show that the long burst from 2S 0918-549 is naturally explained by accretion of pure helium at the observed rate of 0.01 of the Eddington accretion rate.

The observed burst energy of $E_{\text {nuc }}=10^{41}$ erg implies an ignition column depth of $y=E_{\text {nuc }}(1+z) / 4 \pi R^{2} Q_{\text {nuc }} \approx$ $7 \times 10^{9} \mathrm{~g} \mathrm{~cm}^{-2}$, where $Q_{\text {nuc }} \approx 1.6 \mathrm{MeV}$ per nucleon is the energy release for helium burning to iron group nuclei, $R$ is the neutron star radius, and $z$ is the gravitational redshift (we assume $R=10 \mathrm{~km}$ and $z=0.31$, appropriate for a $1.4 M_{\odot}$ neutron star). We have calculated the ignition depth for pure helium following the ignition calculations of Cumming \& Bildsten (2000). The calculation involves finding the temperature profile of the accumulating fuel layer, and adjusting the layer thickness until the criterion for unstable ignition is met at the base. Since hydrogen burning is not active for pure helium accretion, the temperature profile of the layer is set by the heat flux emerging from the neutron star crust. We write this heat flux as $\dot{m} Q_{b}$ erg $\mathrm{cm}^{-2} \mathrm{~s}^{-1}$, where $\dot{m}$ is the mass accretion rate

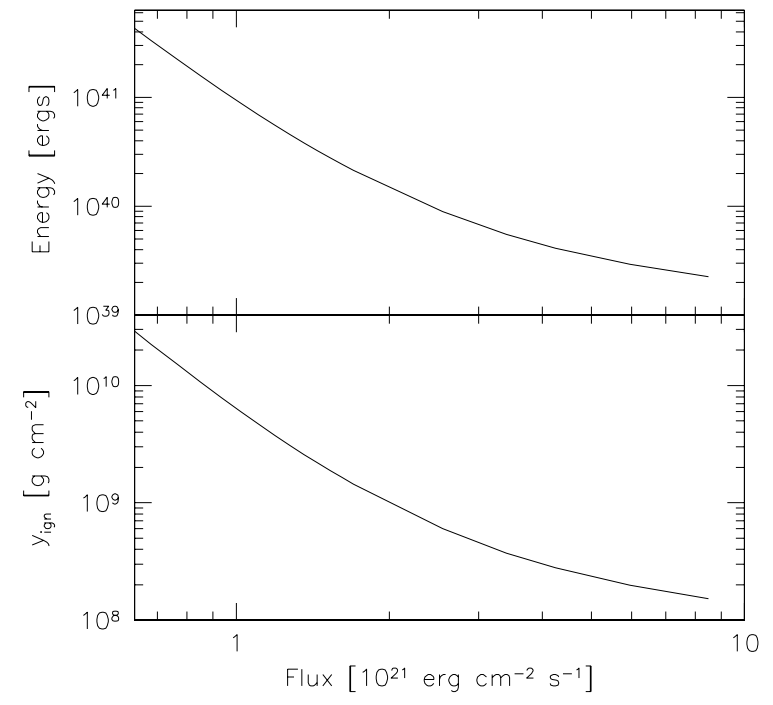

Fig. 8. Ignition thicknesses and flash energies as a function of heat flux.

per unit area, and $Q_{b}$ is the energy per gram released in the crust by pycnonuclear reactions that flows outwards. For low $\dot{m}$, Brown (2000) found that almost all of the $\approx 1.4 \mathrm{MeV}$ per nucleon released in the crust comes out through the surface ${ }^{1}$ (see Fig. 11 of Brown 2000). For $Q_{b} \approx 1 \mathrm{MeV}$ per nucleon or $Q_{b} \approx 10^{18} \mathrm{erg} \mathrm{g}^{-1}$, and using the Eddington accretion rate $\dot{m}_{\mathrm{Edd}} \approx 10^{5} \mathrm{~g} \mathrm{~cm}^{-2} \mathrm{~s}^{-1}$, we find $F_{b} \approx 10^{21} \mathrm{erg} \mathrm{cm}^{-2} \mathrm{~s}^{-1}$ for accretion at $\dot{m}=0.01 \dot{m}_{\text {Edd }}$. Figure 8 shows the ignition column depth and predicted burst energy as a function of base flux. For a base flux of $10^{21} \mathrm{erg} \mathrm{cm}^{-2} \mathrm{~s}^{-1}$, we find an ignition column depth of $y \approx 10^{10} \mathrm{~g} \mathrm{~cm}^{-2}$, in good agreement with the value inferred from the burst energetics.

Additional constraints come from the burst lightcurve and the recurrence time. Figure 9 shows the observed lightcurve compared with theoretical cooling models calculated following Cumming \& Macbeth (2004). In these models, the burning is assumed to take place instantaneously, since helium burning is extremely rapid. We then follow the cooling of the hot layer using a time-dependent thermal diffusion code. We show two curves with a total energy release of $10^{41} \mathrm{erg}$, with column depths $7 \times 10^{9}$ and $10^{10} \mathrm{~g} \mathrm{~cm}^{-2}$. The observed decay is well-reproduced by these models. Unfortunately, the recurrence time of the long burst is not well constrained by observations although the suggestion is that it is long. The expected recurrence time from the ignition models is $y / \dot{m}=$ 116 days $\left(y / 10^{10} \mathrm{~g} \mathrm{~cm}^{-2}\right)\left(\dot{m} / 10^{3} \mathrm{~g} \mathrm{~cm}^{-2} \mathrm{~s}^{-1}\right)^{-1}$. 2S 0918-549 was almost continuously observed with the WFCs for 4 days prior to the burst, but there were two data gaps so that the lower limit to the recurrence time is only 1.1 days. In the $87.3 \mathrm{~d}$ period prior to the long burst, $15.6 \mathrm{~d}$ of effective exposure time were collected with no burst detections. The only other X-ray experiment with coverage of 2S 0918-549 in 1996 is the AllSky Monitor on RXTE, also without burst detections.

\footnotetext{
${ }^{1}$ We expect that the value of $Q_{b}$ will depend on the thermal properties of the neutron star interior, for example, the core temperature and crust thermal conductivity. We will investigate the dependence of the ignition conditions on these factors in a future paper.
} 


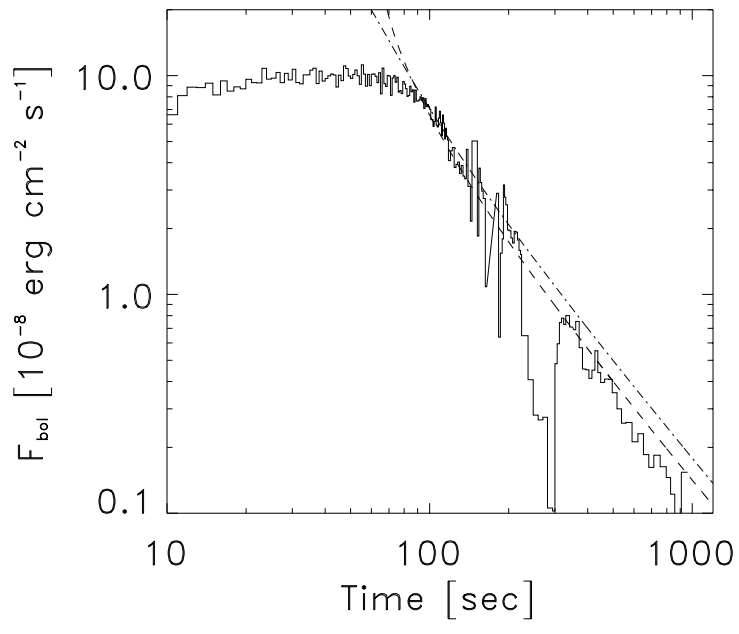

Fig. 9. Comparison of the observed decay of the bolometric black body flux (histogram) with a theoretical model (Cumming \& Macbeth 2004) for the cooling rate of a column of depth $7 \times 10^{9} \mathrm{~g} \mathrm{~cm}^{-2}$ and a nuclear energy release of $1.6 \times 10^{18} \mathrm{erg} \mathrm{g}^{-1}$ which is expected for helium burning to iron (dashed curve). To illustrate the dependence on these two parameters a model is shown with the same energy output $\left(10^{41} \mathrm{erg}\right)$ but for a column depth of $1 \times 10^{10} \mathrm{~g} \mathrm{~cm}^{-2}$ and an energy release of $1.0 \times 10^{18} \mathrm{erg} \mathrm{g}^{-1}$ (dashed-dotted curve).

We have assumed that the accreted material is pure helium. If the accreted material contains carbon, carbon ignition is also possible. However, the burst energy in that case would be $\gg 10^{42}$ erg, and recurrence time $>10$ years (Cumming \& Bildsten 2001). If hydrogen is included in the accreted material, additional heating arises because of CNO burning. Our steadystate accumulation models are only applicable if the temperature is large enough during accumulation $\left(\gtrsim 8 \times 10^{7} \mathrm{~K}\right)$ that the temperature-independent hot $\mathrm{CNO}$ cycle operates. Assuming this to be the case, we find that accretion of solar composition material at a rate $10^{3} \mathrm{~g} \mathrm{~cm}^{-2} \mathrm{~s}^{-1}$ gives an ignition depth smaller than the pure helium case, $6 \times 10^{8} \mathrm{~g} \mathrm{~cm}^{-2}$, and energy release $\approx 10^{40}$ erg (in this model, only the upper $10 \%$ by mass of the layer contains hydrogen). In fact, depending on the accretion history, $\mathrm{CNO}$ burning may be much less than the hot CNO value. Narayan \& Heyl (2003) calculate steady-state models with detailed CNO burning, and find ignition column depths of $\approx 3 \times 10^{9} \mathrm{~g} \mathrm{~cm}^{-2}$ for $\dot{m}=0.01 \dot{m}_{\text {Edd. }}$. Therefore, the burst properties may be similar to those observed if some hydrogen is present. However, we do not expect this because of the likely ultracompact nature of this source.

If the long burst is indeed a helium burst and the other bursts are as well, then the widely varying burst duration must be directly related to the layer thickness. The ignition condition predicts that the ignition thickness decreases with increasing temperature. This implies that the temperature increases from burst 1 to 4 and then decreases again. If the hot CNO cycle is not active, the heat flux from the core/crust and the composition of the outer $100 \mathrm{~m}$ of the NS determines the temperature (Brown et al. 2002). Since the time scale of variation of the crust temperature is expected to be much longer than the burst interval time (years rather than months), the suggestion is there that the composition of the layer changes between bursts.
Given the limited accuracy of our measurements we are unable to test this quantitatively. Temperature variability of roughly a factor of 2 to 3 would be needed to explain a variety of burst durations of a factor of 10 to 100 . The ASM data suggest that the accretion rate shows more variability during the six shorter bursts. Perhaps this explains the earlier ignition and presence of shorter (less energetic) bursts, much as was observed in KS 1731-260 by Cornelisse (et al. 2003, 2004).

\subsection{Evolutionary considerations}

Since the surface layers of the donor are the source of the matter flowing through the accretion disk onto the neutron star, we may conclude that these surface layers are deficient in hydrogen (from the optical spectrum of the disk and the presumed ultracompact nature), do contain helium (from the X-ray bursts), and have an enhanced $\mathrm{Ne} / \mathrm{O}$ abundance ratio (from the X-ray spectrum). This information is useful to discriminate between different evolutionary scenarios.

The evolutionary path of a binary in which a neutron star accretes matter from a companion depends to a large extent on the evolutionary state of the donor at the moment at which mass transfer starts. If mass transfer starts on the main sequence, the orbit shrinks to a minimum of around 70-80 min, and then expands again. At all times during the evolution, the transferred mass consists mainly of hydrogen. It has been suggested that strong magnetic braking may cause the orbit to shrink even if mass transfer is initiated after the donor has evolved a little beyond the terminal age main sequence (Tutukov et al. 1985). The decrease of the orbital period may then proceed to periods less than 70-80 min before the orbit expands again. Even though the hydrogen contents of the transferred mass drop at the shortest periods, the transferred mass is hydrogen rich throughout the evolution in this case also (see e.g. Tables 1 and 2 in Van der Sluys et al. 2005a). Van der Sluys et al. (2005a,b) show that this path to ultrashort periods demands both very special initial conditions and very strong magnetic braking, and thus is unlikely to be important.

If mass transfer starts during shell burning as the star ascends the giant branch, it will lead to long periods if the mass transfer is stable, and eventually to a wide binary of a neutron star an an undermassive white dwarf (e.g. Webbink et al. 1983). However, if the mass transfer is unstable, a spiral-in may ensue, and lead to a close binary of the core of the giant and the neutron star. Mass transfer is increasingly likely to be unstable if the donor star has higher mass, and is further evolved along the giant branch and hence its envelope is fully convective. The post-spiral-in close binary evolves to even shorter periods through loss of angular momentum via gravitational radiation, which may bring the core of the giant, by then cooled into a white dwarf, into contact with the Roche lobe, after which mass transfer starts again. If the spiralin started with the donor in a phase of hydrogen shell burning, the white dwarf is a helium white dwarf. Donors in a phase of helium and carbon shell burning would lead to carbonoxygen and neon-magnesium-oxygen white dwarfs, respectively. Mass transfer from the white dwarf to the neutron star is 
dynamically unstable if the white dwarf has a mass which is too high. The precise limit is somewhat uncertain, depending on the amount of mass and angular momentum loss, but is probably near $0.4-0.5 M_{\odot}$ (see e.g. Yungelson et al. 2002). This limit excludes neon-magnesium-oxygen white dwarfs as well as the more massive carbon-oxygen white dwarfs. Only lowmass carbon-oxygen white dwarfs and helium white dwarfs are possible stable donors for a neutron star.

Mass transfer in a system with a white dwarf donor is a very strong function of the mass of the white dwarf. Immediately after contact, at a period on the order of a few minutes, the mass transfer is highly super-Eddington, and the white dwarf mass decreases rapidly. The orbit expands, and the donor mass decreases quickly until the binary has a mass transfer rate that is sufficiently low to be sustained for a longer period of time. The shortest orbital period observed for a system with a neutron star and white dwarf donor is $11 \mathrm{~min}$. A rough estimate of the mass of the donor $M$ as a function of orbital period $P_{b}$ can be made by combining the mass-radius relation of a white dwarf with the equation giving the size of the Roche lobe for the less massive star in a binary: this gives $M / M_{\odot} \sim\left(50 \mathrm{~s} / P_{b}\right)$. Thus, the 11 min binary has a donor with mass less than $0.1 M_{\odot}$ (Verbunt 1987), and the other ultrashort period systems with known orbital periods have donors of smaller masses still. This implies that the composition of the mass being transferred to the neutron star in these binaries is that of the innermost material of the initial white dwarf.

We illustrate the compositions of the helium and carbonoxygen white dwarfs by means of the core of a model star of $1.5 M_{\odot}$ in Fig. 10 and Table 3. These compositions were computed with the evolution code of Eggleton (1971, 1972) with updated input physics as described in Pols et al. (1995). We do not expect mass loss to have any effect, because the profiles in the inner core have already been established by the time mass loss becomes important. Mass transfer from the white dwarf donor can cause its mantle to become convective, especially near and beyond the period minimum. However, since the core of the star is very homogeneous (Fig. 10), this has little influence on the surface abundances. The demand that the mass transferred in 2S 0918-549 contains helium is obviously compatible with a donor consisting of the inner $\lesssim 0.1 M_{\odot}$ of an initial helium white dwarf; however, it is not compatible with a donor consisting of the inner $\$ 0.1 M_{\odot}$ of an initial carbonoxygen white dwarf. As shown by Fig. 10 the helium content of the central mass of a carbon-oxygen white dwarf is zero (it is at the minimum allowed for computational stability in the evolution code, $10^{-12}$ ). From this, one would have to conclude that the donor at the onset of mass transfer was a helium white dwarf, rather than a carbon-oxygen white dwarf.

This conclusion is strengthened when we consider the neon and oxygen abundances. In the helium core, the neon abundance is still at the zero-age main-sequence composition of the progenitor star, not affected by nuclear evolution (Fig. 10). The oxygen abundance, however, is lower in the helium core as oxygen is converted into nitrogen in the CNO cycle (Iben 1967). The depletion of oxygen is stronger in more massive progenitors, because the CNO cycle takes place at higher temperatures (see Table 4). Thus the $\mathrm{Ne} / \mathrm{O}$ abundance ratio is predicted to

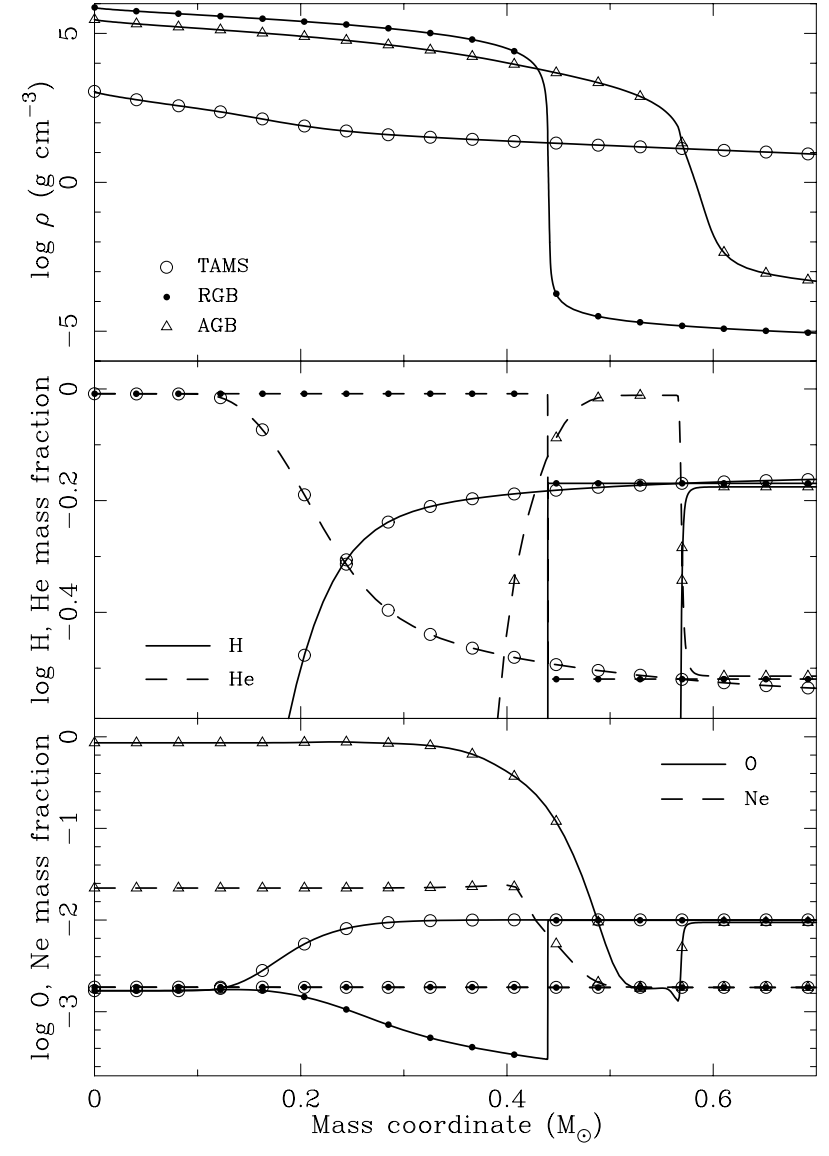

Fig. 10. Three structure plots for different moments in the evolution of a $1.5 M_{\odot}$ model. Only the central $0.7 M_{\odot}$ is shown, as a function of the mass coordinate. Upper panel a): Logarithm of the mass density. Middle panel $\mathbf{b}$ ): Logarithm of the hydrogen (solid lines) and helium (dashed lines) mass fraction . Lower panel c): Logarithm of the oxygen (solid lines) and neon (dashed lines) mass fraction. The symbols on the lines indicate the model, as shown in the upper panel.

be high if the donor in $2 \mathrm{~S} 0918-549$ consists of the central mass of a helium white dwarf. We point out that there is no observational evidence that the mass fraction of neon is enhanced since a complete measurement of the abundance of all expected elements, particularly helium and carbon, is lacking; only the abundance ratios $\mathrm{Ne} / \mathrm{O}$ and $\mathrm{Ne} / \mathrm{Fe}$ have been measured (Juett et al. 2003). Neither is there conclusive observational evidence for an increased oxygen abundance, even when considering the optical spectrum which does suggest the presence of oxygen (and carbon) lines but with insufficient significance to prove the presence (Nelemans et al. 2004).

In a carbon-oxygen white dwarf both neon and oxygen are more abundant, but oxygen more so than neon (Fig. 10). The increase in neon abundance is caused by the conversion of ${ }^{14} \mathrm{~N}$, produced by the $\mathrm{CNO}$ cycle, into ${ }^{22} \mathrm{Ne}$ during core $\mathrm{He}-$ burning ${ }^{2}$. However, this is dwarfed by the production of oxygen

2 The nuclear network in the evolution code does not follow the ${ }^{22} \mathrm{Ne}$ abundance directly, but instead the burnt ${ }^{14} \mathrm{~N}$ is added to the ${ }^{20} \mathrm{Ne}$ abundance assuming particle number conservation (see Pols et al. 1995). The total neon mass fraction we find is therefore slightly underestimated. 
Table 3. Properties of the $1.5 M_{\odot}$ model at the moments the structure plots of Fig. 10 were made. The age is in Gyr, the helium and carbonoxygen core masses in $M_{\odot}$. The last two columns give the mass fraction ratio $\mathrm{Ne} / \mathrm{O}$ in the core, and the ratio of this number to the initial (ZAMS or ISM) $\mathrm{Ne} / \mathrm{O}$ ratio.

\begin{tabular}{lccccc}
\hline \hline Model & Age & $M_{\mathrm{He}}$ & $M_{\mathrm{CO}}$ & $(\mathrm{Ne} / \mathrm{O})_{\mathrm{c}}$ & $(\mathrm{Ne} / \mathrm{O})_{\mathrm{c}} /(\mathrm{Ne} / \mathrm{O})_{\text {in }}$ \\
\hline TAMS & 2.5949 & 0.166 & 0.000 & 1.093 & 5.97 \\
RGB & 2.8495 & 0.440 & 0.000 & 1.093 & 5.97 \\
AGB & 2.9852 & 0.568 & 0.379 & 0.0260 & 0.142 \\
\hline
\end{tabular}

by helium burning. The possibility exists, if the white dwarf has time to cool enough for crystallization to take place, that ${ }^{22} \mathrm{Ne}$ settles in the centre (Yungelson et al. 2002) and reaches there the so-called azeotropic mass fraction, which is between 0.05 and 0.09 (Isern et al. 1991). This is not enough, however, to increase the $\mathrm{Ne} / \mathrm{O}$ ratio above the solar value. Taking into account that the azeotropic $\mathrm{Ne}$ abundance may be underestimated by up to a factor three, the $\mathrm{Ne} / \mathrm{O}$ ratio might be barely reconciled with the observed value, as noted by Yungelson et al. (2002). Nevertheless, if the donor in 2S 0918-549 consists of the central mass of a carbon-oxygen white dwarf the $\mathrm{Ne} / \mathrm{O}$ abundance ratio is expected to be rather low. Conversely, the $\mathrm{Ne} / \mathrm{O}$ ratio observed for $2 \mathrm{~S} 0918-549$ is naturally explained if its donor is the central mass of a helium white dwarf, rather than a carbonoxygen white dwarf.

The helium core denuded by a spiral-in undergoes helium burning if its mass is higher than about $0.34 M_{\odot}$ before it becomes degenerate, which will be the case for stars with initial mass higher than $2.25 M_{\odot}$ (see Table 4 ). In this case a hybrid white dwarf may be formed, with a carbon-oxygen core and a helium mantle. When this white dwarf transfers mass to a neutron star, it will rapidly lose its helium mantle; at orbital periods in excess of 11 min no helium is left.

Thus, a helium white dwarf is the most promising donor in the 2S 0918-549 system. Such a donor is formed when a star with initial mass less than $2.25 M_{\odot}$ enters a spiral-in phase on its first ascent of the giant branch. Based on the work by Deloye \& Bildsten (2003), the observed mass accretion rate predicts for a He WD donor an orbital period between 25 and $30 \mathrm{~min}$. These results are in general agreement with calculations by Belczynski \& Taam (2004) who predict that of all ultracompact binaries with a neutron star accretor, $60 \%$ may have a helium white dwarf donor. Furthermore, our calculations suggest that AM CVn systems, ultracompact binaries in which the accretor is a white dwarf, should show an enhanced $\mathrm{Ne} / \mathrm{O}$ ratio if they have a helium white dwarf companion. Indeed, such an enhancement was recently observed in the AM CVn system GP Com (Strohmayer 2004).

\section{Conclusion}

In conclusion, we have shown that the properties of the long X-ray burst from 2S 0918-549 and the enhanced Ne/O abundance ratio are both consistent with the companion star being a helium white dwarf. This scenario would seem to be at odds with the presence of $\mathrm{C}$ and $\mathrm{O}$ lines and the absence of He lines in the optical spectrum. However, the evidence for
Table 4. Helium core masses and core abundances for model stars with masses between 1.0 and $5.0 M_{\odot}$. The second column gives the range of helium core masses (in $M_{\odot}$ ) that are obtained between the formation of the core and core helium ignition. The third and fourth column give the $\mathrm{Ne} / \mathrm{O}$ abundance of the core, relative to the initial (ISM) $\mathrm{Ne} / \mathrm{O}$ abundance, for the helium core (RGB) and carbon-oxygen core (AGB) respectively.

\begin{tabular}{cccc}
\hline \hline$M$ & $M_{\mathrm{He}-\text { core }}$ & \multicolumn{2}{c}{$(\mathrm{Ne} / \mathrm{O})_{\mathrm{c}} /(\mathrm{Ne} / \mathrm{O})_{\text {in }}$} \\
& & RGB & AGB \\
\hline 1.00 & $0.00-0.47$ & 2.25 & 0.14 \\
1.50 & $0.15-0.47$ & 5.97 & 0.15 \\
2.00 & $0.23-0.39$ & 9.03 & 0.14 \\
2.25 & $0.30-0.34$ & 10.2 & 0.13 \\
2.50 & $0.34-0.36$ & 11.3 & 0.13 \\
3.00 & $0.38-0.42$ & 12.9 & 0.14 \\
4.00 & $0.63-0.64$ & 16.3 & 0.15 \\
5.00 & $0.85-0.85$ & 19.0 & 0.16 \\
\hline
\end{tabular}

$\mathrm{C}$ and $\mathrm{O}$ lines is inconclusive. Nelemans et al. (2004) find that the case is less clear for $2 \mathrm{~S} 0918-549$ than for $4 \mathrm{U} 0614+091$ and $4 \mathrm{U}$ 1543-624 due to the relative faintness of the optical counterpart, and confirmation through deeper observations would be desirable. In addition, non-LTE effects may be important. This is the case in the UV, where Werner et al. (2004) calculated accretion disk models for the UV spectrum and found the He II 1640A line depth to be rather weak even for large helium abundances. Therefore, as yet we regard all X-ray and optical measurements of 2S 0918-549 to be consistent with a helium white dwarf donor star.

Acknowledgements. We thank Ron Remillard for providing the high time-resolution data of the two bursts detected with the RXTE/ASM, and Lars Bildsten, Edward Brown, Deepto Chakrabarty, Duncan Galloway, Peter Jonker, Adrienne Juett and Gijs Nelemans and for useful discussions. J.Z. acknowledges support from the Netherlands Organization for Scientific Research (NWO). A.C. acknowledges support from McGill University startup funds, an NSERC Discovery Grant, Le Fonds Québécois de la Recherche sur la Nature et les Technologies, and the Canadian Institute for Advanced Research. Gerrit Wiersma, Jaap Schuurmans, Nuovo Telespazio and the ASI Science Data Center are thanked for continued support.

\section{References}

Arnaud, K. A. 1996, in Astronomical Data Analysis Software and Systems V, ed. G. Jacoby, \& J. Barnes, ASP Conf. Ser., 101, 17

Belczynski, K., \& Taam, R. E. 2004, ApJ, 603, 690

Bildsten, L., Salpeter, E., \& Wasserman, I. 1992, ApJ, 384, 143

Bildsten, L., Chang, P., \& Paerels, F. 2003, ApJ, 591, L29

Boella, G., Butler, R. C., Perola, G. C., et al. 1997a, A\&AS, 122, 299

Boella G., Chiappetti, L., Conti, G., et al. 1997b, A\&AS, 122, 327

Brandt, S., Castro-Tiradi, A. J., Lund, N., et al. 1992, A\&A, 262, L15

Brandt, S., Lund, N., \& Castro-Tirado, A.J. 1993a, IAUC 5710

Brandt, S., Lund, N., \& Castro-Tirado, A.J. 1993b, IAUC 5717

Brown, E. F. 2000, ApJ, 531, 988

Brown, E. F., Bildsten, L., \& Chang, P. 2002, ApJ, 574, 920

Chevalier, C., \& Ilovaisky, S. A. 1987, A\&A, 172, 167 
Cominsky, L., Forman, W., Jones, C., \& Tananbaum, H. 1977, ApJ, 211, L9

Cominsky, L. 1981, Ph.D. Thesis, MIT

Cornelisse, R., Verbunt, F., In 't Zand, J. J. M., et al. 2002, A\&A, 392, 885

Cornelisse, R., In 't Zand, J. J. M., Verbunt, F., et al. 2003, A\&A, 405, 1033

Cornelisse, R. 2004, Rev. Mex. Astron. Astrofis., 20, 10

Cumming, A., \& Bildsten, L. 2000, ApJ, 544, 453

Cumming, A., \& Bildsten, L. 2001, ApJ, 559, L127

Cumming, A., \& Macbeth, J. 2004, ApJ, 603, L37

Deloye, C. J., \& Bildsten, L. 2003, ApJ, 598, 1217

Eggleton, P. P. 1971, MNRAS, 151, 351

Eggleton, P. P. 1972, MNRAS, 156, 361

Frontera, F., Costa, E., Dal Fiume, D., et al. 1997, A\&AS, 122, 357

Fujimoto, M. Y., Hanawa, T., \& Miyaji, S. 1981, ApJ, 247, 267

Galloway, D. K., Cumming, A., Kuulkers, E., et al. 2004, ApJ, 601, 466

Galloway, D., Muno, M. P., Chakrabarty, D., et al. 2005, in preparation

Harmon, B. A., Wilson, C. A., Fishman, G. J., et al. 2004, ApJS, 154, 585

Hoffman, J. A., Cominsky, L., \& Lewin, W. H. G. 1980, ApJ, 240, L27

Homer, L., Charles, P. A., Naylor, T., et al. 1996, MNRAS, 282, L37

Hua, X.-M., \& Titarchuk, L. 1995, ApJ, 449, 188

Iben, I., Jr. 1967, ApJ, 147, 624

In 't Zand, J. J. M., Verbunt, F., Strohmayer, T. E., et al. 1999, A\&A, 345,100

In 't Zand, J. J. M., Verbunt, D., Kuulkers, E., et al. 2002, A\&A, 389, L43

In 't Zand, J. J. M., Cornelisse, R., \& Cumming, A. 2004a, A\&A, 426, 257

In 't Zand, J. J. M., Cornelisse, R., Kuulkers, E., Verbunt, F., \& Heise, J. 2004b, in Proc. The Restless High-Energy Universe', ed. E. P. J. van den Heuvel, J. J. M. in 't Zand, \& R. A. M. J. Wijers, Nucl. Phys. B, Proc. Suppl. Ser., 132, 657

Isern, J., Hernanz, M., Mochkovitch, R., \& Garcia-Berro, E. 1991, A\&A, 241, L29

Jager R., Mels, W. A., Brinkman, A. C., et al. 1997, A\&AS, 125, 557

Jonker, P. G., van der Klis, M., Homan, J., et al. 2001, ApJ, 553, 335

Juett, A. M., Psaltis, D., \& Chakrabarty, D. 2001, ApJ, 560, L59

Juett, A. M., \& Chakrabarty, D. 2003, ApJ, 599, 498

Juett, A. M., \& Chakrabarty, D. 2005, ApJ, submitted [arXiv: astro-ph/0501472]
Kuulkers, E. 2004, in Proc. The Restless High-Energy Universe, ed. E. P. J. van den Heuvel, J. J. M. in 't Zand, \& R. A. M. J. Wijers, Nucl. Phys. B, Proc. Suppl. Ser., 132, 466

Levine, A., Bradt, H., Cui, W., et al. 1996, ApJ, 469, L33

Morrison, R., \& McCammon, D. 1983, ApJ, 270, 119

Nakagawa, Y. E., Yamazaki, T., Suzuki, M., et al. 2004, in Proc. Gamma-Ray Symposium, Santa Fe, September 8-12, 2003

Narayan, R., \& Heyl, J. S. 2003, ApJ, 599, 419

Nelemans, G., Jonker, P. G., Marsh, T. R., \& van der Klis, M. 2004, MNRAS, 348, L7

Nelson, L. A., Rappaport, S. A., \& Joss, P. C. 1986, ApJ, 304, 231

Parmar, A. N., Martins, D. D. E., Bavdaz, M., et al. 1997, A\&AS, 122, 309

Pols, O. R., Tout, C. A., Eggleton, P. P., \& Zhanwen, H. 1995, MNRAS, 274, 964

Strohmayer, T. E. 2004, ApJ, 608, L53

Strohmayer, T. E., \& Brown, E. F. 2002, ApJ, 566, 1045

Swank, J. H., Becker, R. H., Pravdo, S. H., Saba, J. R., \& Serlemitsos, P. J. 1976, IAUC 3010

Swank, J., Becker, R. H., Boldt, E. A., Holt, S. S., \& Serlemitsis, P. J. 1978, MNRAS, 182, 349

Titarchuk, L. 1994, ApJ, 434, 313

Titarchuk, L., \& Lyubarskij, Y. 1995, ApJ, 450, 876

Tutukov, A. V., Fedorova, A. V., Ergma, E. V., \& Yungelson, L. R. 1985, Sov. Astron. Lett., 11, 52

Ubertini, P., Bazzano, A., Cocchi, M., et al. 1999, ApJ, 514, L27

Van der Sluys, M.V., Verbunt, F., \& Pols, O. R. 2005a, A\&A, 431, 647

Van der Sluys, M.V., Verbunt, F., \& Pols, O. R. 2005b, A\&A, submitted

van Paradijs, J. 1996, A\&A, 464, L139

Van Paradijs, J., \& McClintock, J. E. 1994, A\&A, 290, 133

Verbunt, F. 1987, ApJ, 312, L23

Wallace, R. K., \& Woosley, S. E. 1981, ApJS, 45, 389

Wang, Z., \& Chakrabarty, D. 2004, ApJ, 616, L139

Webbink, R. F., \& Rappaport, S., \& Savonije, G. J. 1983, ApJ, 270, 678

Werner, K., Nagel, T., Dreizler, S., \& Rauch, T. 2004, Rev. Mex. Astron. Astrofis., 20, 146

White, N. E., Kaluzienski, J. L., \& Swank, J. H. 1984, in High Energy Transients in Astrophysics, ed. S. E. Woosley, AIP Conf. Proc., 115,31

Yungelson, L. R., Nelemans, G., \& van den Heuvel, E. P. J. 2002, A\&A, 388, 546 